\title{
Analysis of patterns formed by two-component diffusion limited aggregation
}

\author{
E. B. Postnikov* \\ Kursk State University, Department of Theoretical Physics, Radishcheva st., 33, 305000 Kursk, Russia
}

A. B. Ryabovit

University of Oldenburg, ICBM, 26111 Oldenburg, Germany

\author{
A. Loskutov \\ Moscow State University, Physics Faculty, 119992 Moscow, Russia
}

\begin{abstract}
We consider diffusion limited aggregation of particles of two different kinds. It is assumed that a particle of one kind may adhere only to another particle of the same kind. The particles aggregate on a linear substrate which consists of periodically or randomly placed particles of different kinds. We analyze the influence of initial patterns on the structure of growing clusters. It is shown that at small distances from the substrate, the cluster structures repeat initial patterns. However, starting from a critical distance the initial periodicity is abruptly lost, and the particle distribution tends to a random one. An approach describing the evolution of the number of branches is proposed. Our calculations show that the initial patter can be detected only at the distance which is not larger than approximately one and a half of the characteristic pattern size.

PACS numbers: $61.43 . \mathrm{Hv}$
\end{abstract}

\section{INTRODUCTION}

It is well known that diffusion limited aggregation (DLA), firstly proposed by Witten and Sander [1], is a quite suitable model of a number of physical phenomena. For example, it describes kinetic processes of the growth of electro-deposited dendrites, colonies of bacteria, viscous fingers in fluid mixtures, etc. Such a variety of applications induced theoretical and numerical analysis of DLA, and at present it is a well-studied model which has been verified on various theoretical and experimental levels (from the mean-field approach to the detailed microscopic representation). A comprehensive review can be found, for instance, in the book [2].

Generalization of DLA can provide a proper description of more complex pattern formation processes (see, e.g., [3, 4]). This interest was inspired by the emergence of micro- and nanoscale structures, which are built by deposition of diffusing particles on a surface. In particular, the authors of the paper [5] experimentally studied layer-by-layer deposition and investigated the cluster structure in each layer. Moreover, there are important biophysical and biotechnological examples: the formation of self-assembled patterns such as biomimetics and biominerals [6, 7]; technologies of fabrication and usage of templates for the cell/protein aggregate formation [8-10], etc. Finally, the formation of clusters on a given pattern can serve as a tool for the amplification of scattered or emitted signals [11].

A natural generalization of the classical DLA is a mul-

\footnotetext{
*Electronic address: postnicov@gmail.com

${ }^{\dagger}$ Electronic address: a.ryabov@icbm.de

‡Electronic address: loskutov@chaos.phys.msu.ru
}

ticomponent cluster formation. In this case the growing structure is created from distinct kinds of particles which interact with each other in different ways. For the multicomponent DLA, random deposition of particles on the growing cluster changes the particle distribution in layers so that the initial pattern becomes more and more fuzzy with the distance from the initial germ. This induces questions about the influence of the deposition on the structure of layers and about the competing roles of the growth and diffusion processes in the formation of multilayer clusters.

The DLA-model of two non-interacting kinds of particles was advanced in the paper 12. The authors showed that the branches of two kinds can coexist and unrestrictedly grow if and only if the particles of different kinds appear in the system with the equal probability. A more detailed description of the aggregation of various kinds of particles was proposed in [13, 14], where it was argued that if the branches of various kinds are impenetrable then the structure of aggregates is completely determined by initial fluctuations. Recently it has been shown [15] that the fractal dimension of a multicomponent DLA-cluster can be found by means of the coarsegrained mean-field approach. This method allows correct estimations of the fractal dimension even if we neglect the fluctuations in the distribution of particles.

In the present paper we consider two-component DLA on a substrate (germ) which consists of periodically or randomly distributed particles. We assume that new particles of both kinds arise with the same probability and can aggregate only on the particles of the same kind. The number of initial single-kind patches on the substrate determines the maximal number of branches which can appear in the formed cluster. However, is the substrate contains short patches then in a few neighbor layers initial periodicity is lost, and we observe a random particle 
distribution. Otherwise, if the initial characteristic size of patches is large enough then the pattern periodicity persists until a certain layer. In the further layers the periodic structure becomes more and more fuzzy and the particle distribution tends to a random one.

To analyze the scaling properties of this process we used two characteristics: the number of branches at a certain distance from the substrate and the spectrum of the wavelet transform with Haar basis. Both approaches show self-similarity of the clusters. The wavelet analysis shows that at the distance larger than approximately $3 / 2$ of the initial patch size the branch distribution becomes indistinguishable from a random one.

\section{THE MODEL}

As a model of layer-by-layer random deposition on a given initial matrix, we consider a two-component offlattice DLA process in cylindrical geometry. Particles of two non-interacting kinds (marked as grey and black (red and black in color online version), see Fig. 1) randomly walk and aggregate on initial substrate in a rectangular area of height $H$ and width $W$ with lateral periodic boundary conditions.

An initial pattern (Fig. 1A) is formed by particles of two kinds, which are periodically or randomly distributed along the bottom side of the rectangle. A new particle of a randomly chosen kind appears at the upper side of the rectangular area and diffuses within it. If this particle leaves the area across the upper side, then we generate a new one. Colliding with a seed of another kind, the particle is reflected and proceeds its motion. Upon a collision with a seed of the same kind, it sticks and becomes a part of the cluster. After aggregation a new particle appears at the upper side and so on. As a result, branches of different kinds grow on the initial substrate (Figs. 1B-1D). To avoid the domination of particles of one kind [12], we assume that the appearance of particles of every kind is equiprobable.

In our simulation, the height $H$ of the rectangle is equal to the fourfold maximal cluster height (which is 500 particle diameters), and the width $W$ is equal to 4096 particle diameters. Also, to enhance our simulations we used a few improvements of the algorithm. Firstly, the particle wandering within a "fjord" composed of particles of another kind may dramatically increase the simulation time. To avoid this we limit the particle lifetime by 50000 steps. Secondly, we dynamically change the step size of the random walk, increasing it far from clusters and reducing it up to 0.1 of the particle diameter in the vicinity of clusters [16]. To control the finite size effects we repeated the same simulations on two times shorter substrates $(W=2048)$. These experiments show the same results until the number of cluster brunches is larger than 10. Thus before this threshold the influence of finite size effects is negligible.

To analyze the evolution of the branch distributions we
A

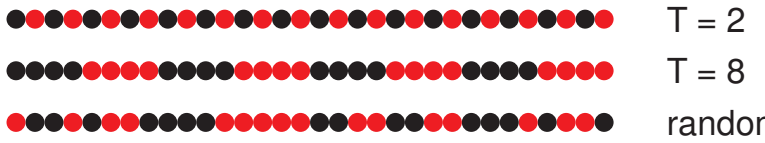

B

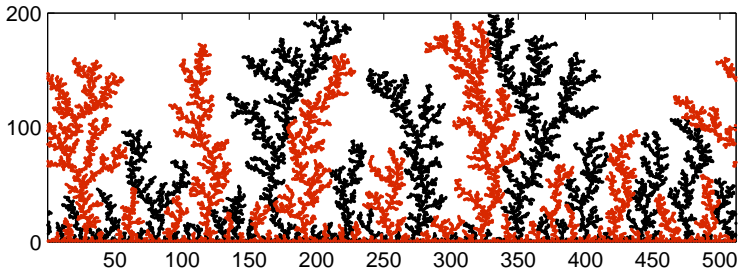

C

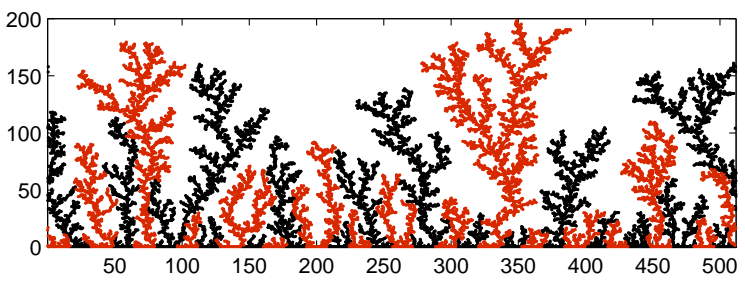

D

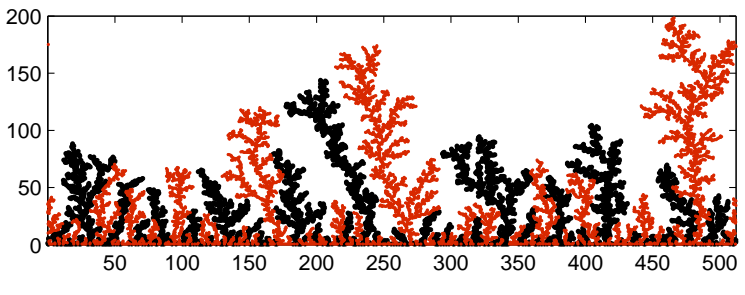

Figure 1: (Color online) (A) — Periodically and randomly assembled substrates of particles of two non-interacting kinds (grey and black/red and black - in color online version). (B), (C), and (D) - Typical DLA-clusters on different substrates: $T=2, T=32$, and random initial distribution, respectively. To be more visible, width $W$ of the initial substrate consists of 512 particles (i.e. $1 / 8$ used for numerical simulations.

simulate the growth of clusters on random and periodical substrates (Fig. 1A). In the former case particles of both kinds are initially randomly distributed on the line. In the latter case, they are placed periodically so that $N$ particles of one kind alternate with $N$ particles of another kind. Thus, the period of such patterns is $T=2 N$. We perform simulations for the periods $T=2,4,8,16,32$, and $T=64$ particles.

The limitation in the period length $(T \leq 64)$ is determined by the finite size of substrates and periodic boundary conditions. This periodicity implies that the actual number of branches will always be integer.

Thus our results are valid until the number of branches is essentially larger than one. In fact, see below, it is possible to neglect the boundary effects until the cluster includes at least 10 branches.

For numerical analysis, we discretized the distance 
from the substrate using the particle diameter as a minimal unit of length. Thus, we define a set of horizontal layers with the height of one unit, which are numbered in accordance with their distance from the substrate. The substrate is located in the first layer.

\section{CLUSTER MORPHOLOGY}

Apparently, with an increase of the distance from the substrate, the influence of initial patterns vanishes, and the initially periodic branch distribution becomes more and more random. Figs. 1B-1D show that the initial size of mono-color patches on the substrate plays a crucial role for the cluster morphology. If a substrate has a short period $(T \leq 4)$ then the initial periodic structure is rapidly lost. In fact, for $T=2$ the distribution of colors appears to be random already in the second layer. However, if $T \geq 8$ then a DLA-cluster can develop inside of each mono-color region, preserving its initial periodicity until a certain layer.

The average mass of cluster layers gives a rough characteristic of clusters on different initial substrates. On random and short-periodic $(T \leq 4)$ substrates the mass of layers decays as a power law $M(n) \propto n^{\alpha_{m}}$, where $\alpha_{m}=-0.39$, thereby resulting in the fractal dimension $D \simeq 1.61$ of these clusters.

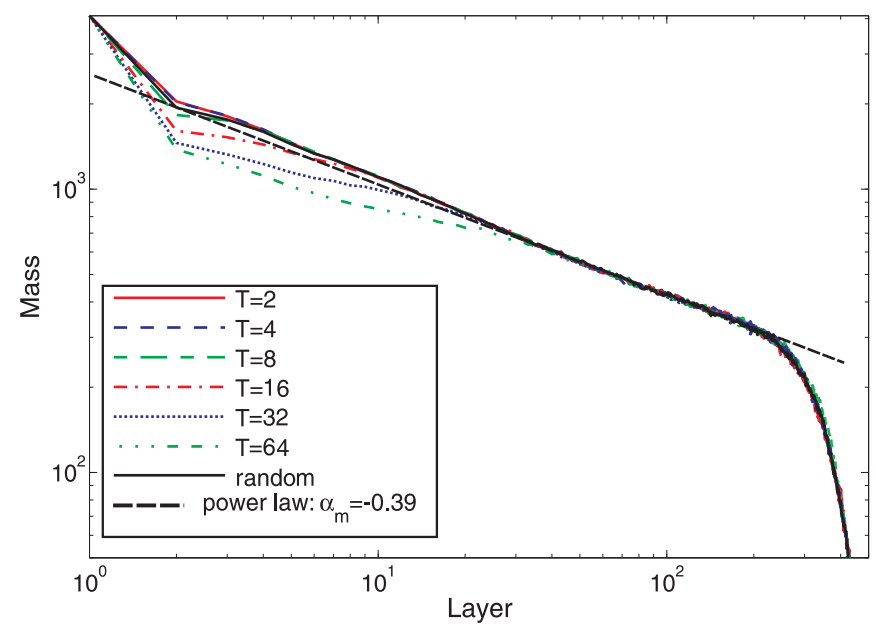

Figure 2: (Color online) The layer mass as a function of the layer number for clusters growing on periodically and randomly assembled substrates.

This value is close to the fractal dimension of multicomponent DLA clusters in radial geometry [15]. As shown in this article, an additional screening by branches of different kinds decreases the fractal dimension in comparison with the standard value 1.78 .

By contrast, the long-periodic $(T \geq 8)$ substrates lead to sparser aggregates. The main reason is that every mono-color patch on the substrate gives rise to a single DLA branch which, attaching almost all particles of the same kind, shades its base. Therefore, the wider the initial mono-color patches, the smaller the density of the cluster arising on this substrate. Note that from a certain layer the layer mass approaches that obtained for randomly assembled substrates (black line in Fig. 2).

More information about the cluster morphology we can obtain evaluating the number of different branches. For instance, in radial geometry this number increases with the distance from the cluster center [17]. By contrast in cylinder geometry, the number of branches decreases with the layer number, because branches of the same kind can merge, stopping growth of other branches squeezed between them. To evaluate the number of branches, we trace the colors of particles along each layer and register changes of the colors. Thus, we combine two branches of the same kind into one if there is no branch of another kind between them in a certain layer. In other words, we assume that these branches merge. This procedure is correct in the layers where the aggregation has already stopped, however it underestimates the number of branches for those regions of cluster which actively adsorb new particles.

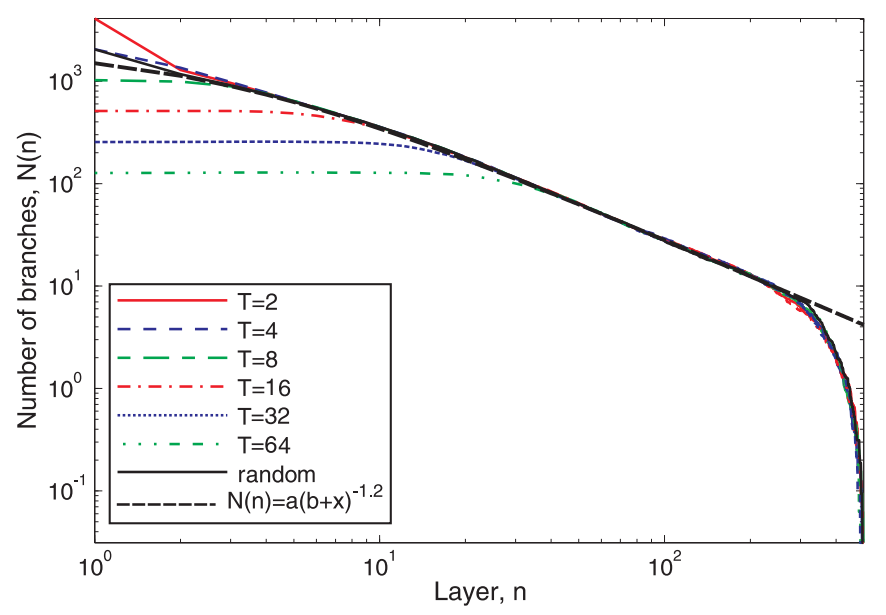

Figure 3: (Color online) The number of branches as a function of the layer number in clusters growing on periodically and randomly assembled substrates. The black dashed line shows the power low $N(n)=a(b+x)^{-1.2}$, where $a=7288, b=2.74$.

Fig. [3] shows the average number of branches as a function of the layer number. As expected, for all initial distributions this value decreases. However, we want to highlight several aspects. Firstly, all curves finally approach the curve obtained on the random substrate. The number of branches, $N$ decreases with the layer number, following the power low $N(n) \propto(n+b)^{-1.2}$. The initial shift $b$ probably occurs because initially the particles are distributed without gaps between them and the transition to a sparse structure takes a few layers.

Secondly, the numbers of branches on a random and on a periodic with $T=4$ substrate almost coincide. This coincidence apparently arises from the fact that initially these substrates have the same average number (1024) of different mono-color patches (see Appendix A). Thirdly, 
if a substrate has more patches than a random one, then the number of branches abruptly decreases up to the characteristic value obtained on the random substrate.

For instance, if $T=2$ then each patch consists of a single particle and the number of branches decreases threefold already in the second layer. Aggregates on substrates with a large initial period $(T \geq 8)$ have less branches and preserve their periodicity approximately until they have as many branches as on a random substrate. After this layer, the branches begin to extinct and their total number decreases and coincides with the number of branches on randomly assembled substrates. Therefore, the number of branches growing on a random substrate defines a natural limit, and aggregates preserve their initial periodicity as long as they have less branches than clusters growing on a random substrate at the same layer.

\section{WAVELET SPECTRAL ANALYSIS}

The number of branches provides just a rough insight into the cluster structure. More detailed information can be gained from the spectral analysis.

Divide every layer into $W$ square cells of a unit size. In each cell of the layer $n$ we define the piecewise-constant function $f_{n}(x)$ such that $f(x)$ is equal to +1 or -1 if the center of a grey (red in color online version) or black particle, respectively, is located within the cell, and $f_{n}(x)=0$ otherwise. Note that sometimes in our simulations a cell can confine the centers of two particles. To resolve this uncertainty we used the color of the left particle. However, these events rarely occurred, and thus they can not influence the final result [18].

Since the piecewise-constant function $f_{n}(x)$ takes discrete values $\{-1,0,1\}$ on a uniform grid of cells, for the spectral analysis it is natural to use the wavelet transform with Haar orthonormal basis functions

$$
\psi_{j k}(x)=\sqrt{2^{j}} \psi\left(2^{j} x-k\right),
$$

where $j$ and $k$ are the integer numbers, which characterize the scale and the shift, respectively, of Haar wavelet basis function

$$
\psi(x)=\left\{\begin{aligned}
1, & x \in[0,1 / 2) \\
-1, & x \in[1 / 2,1] \\
0, & x \notin[0,1] .
\end{aligned}\right.
$$

Every layer includes $W$ cells. Thus, to represent the function $f_{n}(x)$, the largest wavelet pattern should have the scaling factor $J=\log _{2} W$, and the wavelet expansion of the function $f_{n}(x)$ reads

$$
f_{n}(x)=c_{0}+\sum_{j=0}^{J} \sum_{k=0}^{W-1} d_{j k}^{(n)} \psi_{j k}(x),
$$

where the index $n$ refers to the layer number, $c_{0}$ is the mean value of the function $f_{n}(x)$ and the coefficients

$$
d_{j k}^{(n)}=\int_{0}^{W} f_{n}(x) \psi_{j k}(x) d x
$$

show the partial impact of the wavelet with period $T=2^{j}$ in the sites from $k$ to $k+T$. Thus, the sum of the wavelet components with the same scaling factor,

$$
F_{j}^{(n)}(x)=\sum_{k=0}^{W-1} d_{j k}^{(n)} \psi_{j k}(x),
$$

gives the total impact of wavelets with period $T=2^{j}$ and represents a counterpart of an individual harmonic in the Fourier expansion. Exploiting this analogy, we can refer to the sum

$$
E_{j}^{(n)}=\sum_{k=0}^{W-1}\left(d_{j k}^{(n)}\right)^{2}
$$

as a power of the spectral component including all wavelets with period $T=2^{j}$. Thus, the set of values $\left\{E_{j}{ }^{(n)}\right\}$ where $j=0,1, \ldots, J$ represents the global power spectrum of the wavelet transform of the layer $n$.

The power spectrum of the initial substrates with pe$\operatorname{riod} T$ has only a single non-zero component $E_{j^{*}}{ }^{(1)}$, where $j^{*}=\log _{2} T$. This component represents the main harmonic of the initial wavelet spectrum. Other components appear in the second layer, and their relative impact increases with the layer number, reflecting the transition from the periodic to the random structure of clusters.

However, during this transition the value $E_{j^{*}}^{(1)}$ dominates, thereby reflecting the fact that initial periodicity is preserved in the cluster structure, i.e., the distribution of branches "remembers" the initial distribution.

Consider now the normalized average power of the main harmonics:

$$
\bar{E}_{j^{*}}(n)=\frac{1}{\sqrt{2^{j^{*}-1}} N_{T}}\left\langle E_{j^{*}}^{(n)}\right\rangle,
$$

where $\langle\ldots\rangle$ denotes the average over all realizations. To compare the power of different harmonics on the same scale, we introduced the specific renormalization factor, $1 /\left(\sqrt{2^{j^{*}-1}} N_{T}\right)$. This factor compensates for the differences in the number of initial periods, $N_{T}=W / T$, and in the scales of the basis wavelet functions with various $j^{*}$.

Fig. 4]shows the normalized power of the main harmonics for periodic substrates (color solid lines) in comparison with one harmonic $(T=2)$ on randomly assembled substrates (dash-dotted line).

On randomly assembled substrates the power spectrum equally includes all possible periodic components, which from the outset follow the power law with the slope $\alpha_{w}=0.4$ (see dash-dotted black line in Fig. (4).

Consider how the power of the main harmonics evolves with layer number for substrates which have large initial periodicity: $T \geq 8$. Until the number of branches is less than the number of branches growing on a random substrate (compare with Fig. 33), the power of the main harmonics decreases relatively slow. However, at a critical layer, when this number is exceeded, this decrease 


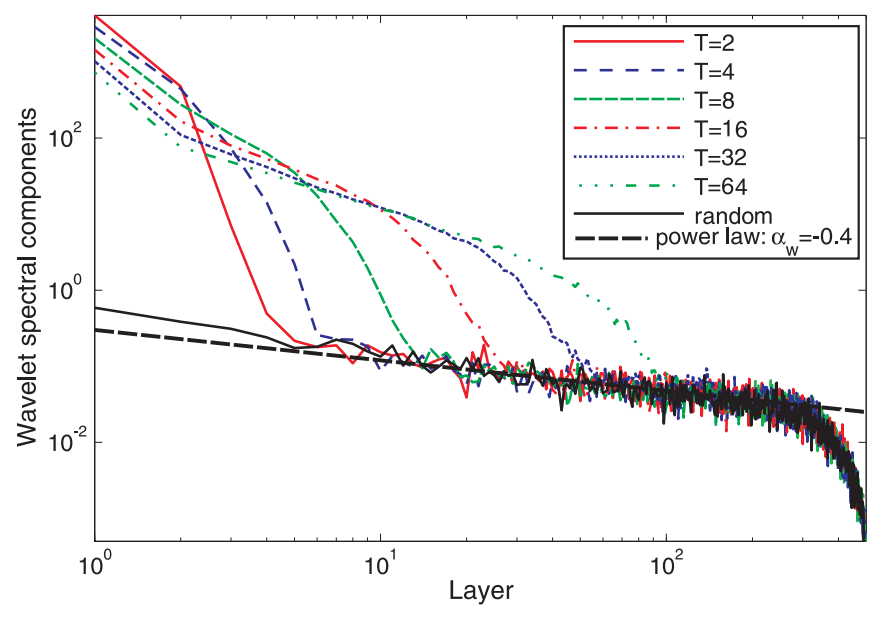

Figure 4: (Color online) The distribution of normalized global power spectrum of wavelet components corresponding to the various initial periods.

becomes much steeper and the power of the main harmonics abruptly declines to the level of other harmonics. Finally, the power of all harmonics goes over the same power law with the exponent $\alpha_{w}=-0.4$.

Note that our approach has a few common traits to the method based on averaged wavelet coefficients, which was suggested in [19] for the analysis of self-affine time series. However, in contrast to the this work, we analyze not the inner structure of a unique function but the set of functions corresponding to a certain layer of a cluster.

Thus, we can select three stages. During the first stage the clusters preserve their initial periodicity and the initial harmonics prevails. During the second stage the main harmonics abruptly decreases and some branches rapidly extinct. In the last stage the distribution of branches becomes indistinguishable from a random distribution. If the initial period is small $(T \leq 4)$ then we observe only the last two stages.

Note that because of the finite cluster height we observe this behavior only approximately within the first 300 layers. Afterwards all harmonics abruptly decay. Furthermore, the substrate width seemingly influences the development of clusters with initial period $T=64$.

\section{MEAN-FIELD ANNIHILATION}

Initially the substrate has an invariance with respect to a shift by one period length. A particle, which randomly attaches to a branch, limits access of other particles to the adjacent branch and breaks the local symmetry. This process is stochastic and local changes accumulate and finally lead to extinction of some branches. A a result, the influence of initial patterns reduces with distance from substrates.

A rough insight into the dynamics of this process can be gained by a mean-field approximation arising from the simple diffusion-annihilation model suggested in [20]. Namely, if two branches of kind $A$ shade a branch of kind $B$ between them, then we can write this as the annihilation process

$$
A_{n}+B_{n}+A_{n}=A_{n+1}
$$

where $n$ is the layer number.

Let $N(n)$ be the average number of branches of the same kind, and let $k$ be the annihilation reaction rate of the branches. The inverse concentration $1 / c$ determines the mean distance between two branches of the same kind. Therefore, the characteristic time after which two branches can merge and shade another branch is equal to $\Delta n \propto 1 / k c$. Furthermore, the decrease of the concentration should be proportional to the density of branches. Therefore, the differential equation of this annihilation process has the form:

$$
\dot{N} \cong \frac{\Delta N}{\Delta n}=-k N^{2} .
$$

Solving this equation we obtain

$$
N(n)=\frac{1}{k}\left(\frac{1}{N(0) k}+n\right)^{-1} .
$$

This solution qualitatively describes the dependence of the number of brunches in Fig. 3, it also predicts the initial shift $b=1 / N(0) k$. Note that the exponent of this dependence is integer, which is common for simple meanfield approximations. However, as the two exponents $(-1$ and -1.2 ) are sufficiently close, this formula gives a good approximation in a certain range.

\section{DISCUSSIONS AND CONCLUSION}

In this paper we analyze the structure of DLA-clusters, growing in a rectangular area with lateral boundary conditions. The clusters are built up by aggregation of particles of two different kinds on random or periodic substrates. Growing DLA branches can merge with each other, stopping the growth of other branches between them. This decreases the number of distinguishable branches and changes the structure of the cluster as a whole. Thus, since this process is stochastic, the influence of initial patterns reduces with distance from substrates.

In more details, we investigated how the rate with which the system loses its initial periodicity depends on the distance from the substrate and showed that there are three characteristics areas. In the first area, which includes first few layers, the average number of clusters preserves and the wavelet harmonics corresponding to the initial periodicity decay slowly. This area exists only if the number of different patches on the substrate is initially smaller than that on a randomly assembled one. In the following layers (where the number of different 


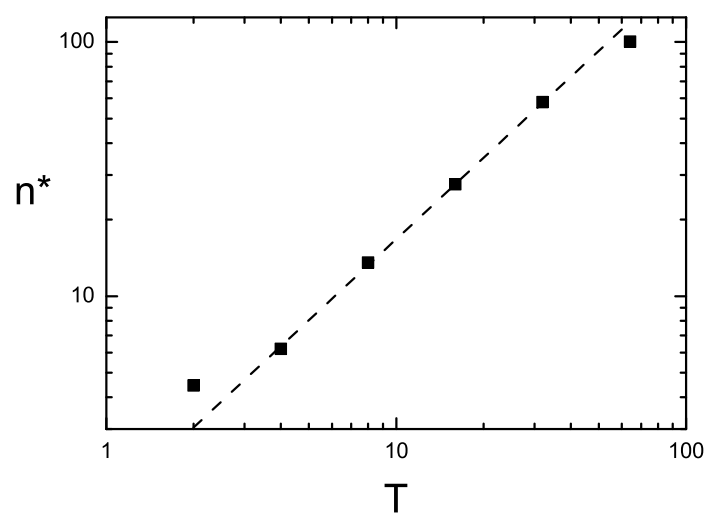

Figure 5: The maximal distance $n^{*}$ from the initial substrate after which the initial periodicity cannot be detected in dependence of the initial period. The black line is fitting $n^{*}=1.46 T^{1.06}$.

branches becomes close to that on a random substrate, or in the second layer if this number was exceeded initially) the initial periodicity drastically decreases and the distribution of branches approaches to a random one. Finally, the average number of branches in a layer coincides with the number of clusters which appear on a randomly assembled substrate, and their distribution becomes random. In this area the evolution of the number of branches can be estimated within the mean-field approach.

From the practical point of view, our results allow to elaborate a criterion for the validation of experimental methods for the selection and amplification of micropatterns via layer-by-layer deposition of specific reagents (see, e.g., 11]). Fig. [ shows that for every initial period of substrates there is a maximal layer number, $n^{*}$, after which the distribution of branches loses initial periodicity and the magnitude of all harmonics becomes equal. Fig. 5 shows that this value scales with the size of initial patterns. For linear fitting we exclude $T=2$, because this distribution loses its regularity almost in the fist layer, and $T=64$, because in this case $n^{*}$ is close to the layers where we observe effects of finite cluster height. All other points follow a power low dependence with the exponent $\gamma=1.06$. Accuracy of our simulations do not allow to confirm that $n^{*}$ grows faster than $T(\gamma>1)$. Thus as an approximate estimation we suggest $n^{*}<3 T / 2$. This dependence shows the principal limit for detection of the substrate structure at a certain distance from the substrate.

\section{Appendix A: Number of branches in random distribution}

Consider a randomly assembled substrate. The sort of a particle in each location is randomly assigned with the probability $1 / 2$. It is evident that the same kind for $n$ successive particles will be assigned with the probability

$$
P_{n}=\frac{1}{2^{n}} .
$$

Thus, the average size of initial clusters of this kind is

$$
S=\sum_{n=1}^{\infty} n P_{n}=\sum_{n=1}^{\infty} \frac{n}{2^{n}}=2 .
$$

Therefore, the average number of clusters on the random substrate should be the same as we have in the periodic pattern, when 2 particles of one kind succeed 2 particles of another kind, i.e. for $T=4$.
[1] T. A. Witten and L. M. Sander, Phys. Rev. Lett. 47, 1400 (1981).

[2] P. Meakin, Fractals, Scaling and Growth Far from Equilibrium (Cambridge University Press, 1998).

[3] H. Röder, E. Hahn, H. Brune, J.-P. Bucher, and K. Kern, Nature 366, 141 (1993).

[4] P. Jensen, A.-L. Barabási, H. Larralde, S. Havlin, and H. E. Stanley, Nature 368, 22 (1994).

[5] F.-J. Meyer zu Heringdorf, M. C. Reuter, and R. M. Tromp, Nature 412, 517 (2001).

[6] M. Murr and D. E. Morse, PNAS 102, 11657 (2005).

[7] H. Imai, Biomineralization I (Springer, 2007), chap. SelfOrganized Formation of Hierarchical Structures.

[8] M. N. Yousaf, B. T. Houseman, and M. Mrksich, PNAS 98, 5992 (2001).

[9] J. Turner, M. L. Becker, X. Li, J.-S. A. Taylor, and K. L. Wooley, Soft Matter 1, 69 (2005).

[10] A. Khademhosseini, R. Langer, J. Borenstein, and J. P. Vacanti, PNAS 103, 2480 (2006).

[11] P. Suci, M. T. Klem, M. Young, and T. Douglas, Soft
Matter 4, 2519 (2008).

[12] T. Nagatani and F. Sagués, Phys. Rev. A 44, 6723 (1991).

[13] V. Tchijov, S. Rodriguez-Romo, and S. Nechaev, JETP Lett. 64, 549 (1996).

[14] S. Rodriguez-Romo, V. Tchijov, O. Ibañuez Orozco, and V. Castaño, Physica A 347, 301 (2005).

[15] E. B. Postnikov, A. B. Ryabov, and A. Loskutov, J. Phys. A 40, 12033 (2007).

[16] The step size of the random walk can be chosen dynamically [21]. According to this approach, if there are no particles within a circular of radius $R$, then the step size equals $R-d$, where $d$ is the particle diameter. Otherwise (to avoid missing the cluster particles) we use the step size of 0.1 of the particle diameter. In both cases the step direction is given by uniformly distributed random numbers $\phi \in[0,2 \pi]$. We also cheked that two- and fourfold change of the minimal step size and the maximal life time of a particle leads us to the same scaling laws.

[17] B. B. Mandelbrot, B. Kol, and A. Aharony, Phys. Rev. 
Lett. 88, 055501 (2002).

[18] This effect is important only when two particles of different colors are located within one cell. However, this event is extremely rare, because usually there is an essential gap between branches of different color. We have detected only two such events in our simulations, therefore their impact is negligible and cannot affect our results.
[19] I. Simonsen, A. Hansen, and O. M. Nes, Phys. Rev. E 58, 2779 (1998).

[20] S. Redner, Nonequilibrium Statistical Mechanics in One Dimension (Cambridge University Press, 1997).

[21] A. Y. Menshutin and L. N. Shchur, Phys. Rev. E 73, 011407 (2006). 